•综述・

\title{
IUCN生态系统红色名录研究进展
}

\author{
谭剑波 ${ }^{1,2}$ 李爱农 ${ }^{1 *}$ 雷光斌 ${ }^{1}$ 陈国科 ${ }^{3}$ 马克平 ${ }^{3}$ \\ 1 (中国科学院成都山地灾害与环境研究所数字山地与遥感应用研究中心, 成都 610041) \\ 2 (中国科学院大学, 北京 100049) \\ 3 (中国科学院植物研究所植被与环境变化国家重点实验室, 北京 100093)
}

\begin{abstract}
摘要: 生态系统红色名录作为物种红色名录的重要补充, 是基于生态系统水平上的生物多样性评估, 对于重要生 物的生境保护至关重要。当前, 世界自然保护联盟(IUCN)将IUCN生态系统红色名录评估方法作为生态系统受威胁 状况评估的正式标准, 广泛应用于全球生态系统的评估, 在生物多样性保护和生态系统管理中发挥着重要作用。 随着IUCN生态系统红色名录评估体系的修订和推广, 其评估标准日趋完善, 但生态系统制图、生态系统崩溃的概 念以及实际操作层面仍面临挑战。本文回顾了生态系统红色名录的发展过程, 归纳了 IUCN生态系统红色名录评估 方案中生境范围退化(标准A)、生境限制分布(标准B)、非生物环境退化(标准C)、生物过程退化(标准D)以及威胁 定量分析(标准E)这5个指标的含义和评估过程, 并围绕IUCN生态系统红色名录实际评估中面临的挑战, 论述了利 用遥感和生态模型可能提供的解决方案。文中指出, 明确生态系统分类体系以及生态系统崩溃等基本概念对生态 系统红色名录评估至关重要; 尺度效应是评估过程的重要影响因素; 多尺度评估是丰富生态系统红色名录空间信 息的潜在方法; 耦合遥感和生态模型等手段共同参与评估, 是未来研究IUCN生态系统红色名录的重要方法。
\end{abstract}

关键词: IUCN生态系统红色名录; 生态系统受威胁状况; 尺度效应; 生物多样性

\section{Research advances and challenges in the IUCN Red List of Ecosystems}

\author{
Jianbo Tan ${ }^{1,2}$, Ainong $\mathrm{Li}^{1^{*}}$, Guangbin $\mathrm{Lei}^{1}$, Guoke Chen ${ }^{3}$, Keping $\mathrm{Ma}^{3}$ \\ 1 Research Center for Digital Mountain and Remote Sensing Application, Institute of Mountain Hazards and Environ- \\ ment, Chinese Academy of Sciences, Chengdu 610041 \\ 2 University of Chinese Academy of Sciences, Beijing 100049 \\ 3 State Key Laboratory of Vegetation and Environmental Change, Institute of Botany, Chinese Academy of Sciences, Bei- \\ jing 100093
}

\begin{abstract}
The Red List of Ecosystems is a growing toolbox for assessing risks to biodiversity at the ecosystem level, which is complementary to the Red List of Threatened Species and important for the protection of key species' habitats. The IUCN Red List of Ecosystems Criteria was adopted as an official global standard for assessing the risks to ecosystems by the IUCN Council. With the revision and extension of the IUCN Red List of Ecosystems Criteria, this framework is gradually improved, which plays an important role in biodiversity conservation and ecosystem management. However, it faces challenges in ecosystem mapping, the concepts of ecosystem collapse and practical application. This paper reviews the development of the Red List of Ecosystems and introduces the five criterions in the IUCN Red List of Ecosystems protocol (declining in distribution, restricted distribution, abiotic degradation, biotic degradation and quantitative estimates of risk of ecosystem collapse) and how it is used. While focusing on the challenges in the assessment with the IUCN Red List of Ecosystems Criteria, we also discuss the potential solutions to these problems based on remote sensing and ecological models. We points out that the explicit definition of ecosystem collapse and the classification system of ecosystems is essential for applying the IUCN Red List of Ecosystems Criteria; the scale effects have great impacts on the results of assessment; assessment at hierarchical scales may be a potential method to provide spatial information for the IUCN Red List of Ecosystems; incorporating remote sensing
\end{abstract}

收稿日期: 2016-05-15; 接受日期: 2017-02-27

基金项目: 中国科学院委托研究与专项咨询服务课题(KFJ-EW-STS-020-02)、国家自然科学基金(41571373; 41631180)、中国科学院战略先导性科技专 项-碳专项(XDA05050105)和环保部“生态十年”专项(STSN-01-04)

* 通讯作者 Author for correspondence. E-mail: ainongli@imde.ac.cn 
and ecological models into this framework is an important way to study the IUCN Red List of Ecosystems Criteria in the near future.

Key words: IUCN Red List of Ecosystems; threatened status of ecosystem; scale effects; biodiversity

生物多样性作为地球生命系统的物质基础, 直 接为人类和其他生物提供生活必需品, 并通过发挥 相关的生态功能, 间接为地球生物提供适宜的栖息 环境以及各种服务, 具有巨大的研究价值(MA, 2005; Cardinale et al, 2012)。由于全球人口大幅度增 加, 人类向自然索取的物质和服务超过了以往任何 一个时期, 加上全球气候变化的影响, 导致全球范 围内的生物多样性水平大幅度下降(MA，2005; Pimm et al, 2014)。生物多样性的丧失进一步影响 到人类福祉和生态系统健康, 并成为当今重要的 全球问题(Ceballos et al, 2015), 引起国际社会的广 泛关注。

生物多样性评估是制定保护措施的前提, 如何 评估生物多样性已成为研究的热点(Nicholson et al, 2009)。当前的生物多样性的评估大多聚焦在物种水 平上, 并在全球生物多样性的保护和规划中发挥了 重要作用(Pimm et al, 1995; Myers et al, 2000)。随着 研究的深入, 人们发现基于物种水平的评估无法反 映生物多样性所有组分(马克平和钱迎倩, 1998; 陈 国科和马克平, 2012), 而结合宏观尺度共同评估区 域生物多样性水平更加合理。原因主要有以下3个 方面: (1)宏观尺度的参与, 辅助大区域生物多样性 的评估, 可节省人力、物力和财力(Rodríguez et al, 2006); (2)微观和宏观角度的结合, 可综合反映区域 的生物多样性水平(Rodríguez et al, 2012); (3)可以 综合指导区域生物多样性保护措施, 方便科学管理 和决策(Noss, 1996; Ferrier, 2002)。生态系统作为研 究生物与环境相互关系的基本功能单元(Odum, 1977), 是生物学和生态学研究的重要宏观水平 (Noss, 1990)。随着对生物多样性和生态系统认识的 不断加深, 相关学者逐渐认识到生物多样性是生态 系统功能和服务多样性的基础, 稳定、复杂的生态 系统其生物多样性水平更高(Hooper et al, 2005; Hector \& Bagchi, 2007)。千年生态系统评估结果表 明生态系统退化是导致全球生物多样性降低的主 要原因之一(MA, 2005)。生态系统在生物多样性评 估中的作用也得到《生物多样性公约》的认可, 受
到广泛关注(马克平, 2016), 并成为评估全球生物多 样性水平的重要手段之一(马克平, 2013; Rodríguez et al, 2015)。

IUCN生态系统红色名录评估标准是基于生态 系统水平上的生物多样性评估框架, 被世界自然保 护联盟(International Union for Conservation of Nature, IUCN)采纳为评估生态系统受威胁状况的正式 标准(Rodríguez et al, 2012)。该标准旨在监测生态系 统状况, 评估生物多样性面临的风险, 识别受威胁 的生态系统, 为管理和决策提供支撑(Bland et al, 2015)。它通过选取适当的指标，定量表征生态系统 受威胁状态, 并结合相关阈值对各评估指标进行分 级, 确定各生态系统受威胁的等级(Rodríguez et al, 2015)。IUCN生态系统红色名录评估标准已应用于 生态系统状况的监测、优先保护区域的确定、保护 成效的评估以及受威胁生态系统的保护与修复, 并 与物种红色名录相结合, 综合评估区域生物多样性 水平, 为生物多样性资源管理提供服务(朱超等, 2015)。

尽管IUCN生态系统红色名录评估标准经过几 次修订, 已发展到Version 2.0版本(Rodríguez et al, 2015), 但无论在生态系统制图、实际操作层面还是 概念上依然面临较大挑战(Boitani et al, 2015; Keith et al, 2015)。本文回顾了生态系统红色名录的发展 过程, 介绍了 IUCN生态系统红色名录评估框架、实 际评估中面临的挑战以及可能的解决方案, 并探讨 了今后的发展趋势, 拟为我国生态系统红色名录工 作的开展以及完善IUCN生态系统红色名录评估体 系提供参考。

\section{1 生态系统红色名录评估发展进程}

生态系统红色名录于1996年由Edward Maltby 在第一次世界自然保护大会上提出(Rodríguez et al, 2012), 旨在开展生态系统受威胁状况评估, 构建类 似物种红色名录的受威胁生态系统目录。作为物种 生物多样性评估的重要补充, 许多学者从不同角度 开展了生态系统红色名录评估的研究工作, 构建了 
一系列的评估方法(Nicholson et al, 2009)。

生态系统受威胁状况评估源于生态系统健康 和风险评估。生态系统健康和风险评估体系主要研 究生态系统对胁迫的响应, 评估生态系统的健康状 况和面临的风险(Barrett, 1978)。该评估体系通过与 健康生态系统对比, 解析生态系统受胁迫后表现出 的症状, 评估其受威胁的强度和范围(Odum, 1985; Rapport et al, 1998)。美国环境保护署基于大量的样 地调查数据, 利用该方法评估了样地区域的生态风 险和健康状况(Lackey, 1994)。该评估体系主要侧重 于相关概念和过程的分析, 为生态系统威胁评估提 供相关的理论基础, 但是需要大量的地面调查数据, 评估难度较大, 很难在大范围区域进行。

随着研究的深入, 研究人员发现生境面积越小, 生态系统的结构和功能越单一, 生物多样性水平越 低, 受威胁的风险越高 (Driver, 2005; Hector \& Bagchi, 2007)。在该理论的推动下, 自1990年以来, 许多国家和地区基于生物多样性与生境面积、生态 系统的结构和功能之间的关系开展了生态系统受 威胁状况评估工作。例如, 《德国受威胁生境红色 名录》(Blab et al, 1995)、《澳大利亚环境保护和生 物多样性法案》(Commonwealth of Australia, 1999)、 《南非受威胁生态系统国家名录》(Rouget et al, 2004)、《非洲生物多样性优先保护区域名录》 (Burgess et al, 2006)、《新西兰陆地环境评估》 (Walker et al, 2006)、《挪威生态系统红色名录》 (Lindgaard \& Henriksen, 2011)等都是较为成熟的具 有代表性的案例。其中《德国受威胁生境红色名录》 首次提出利用生态功能区作为评估单元, 澳大利亚 将评估结果应用于生物多样性保护法案的制订, 南 非利用植被图和土地覆被图作为评估单元, 非洲优 先保护区域的评估采用专家打分系统, 新西兰利用 环境因子与生态系统的关系开展受威胁评估, 《挪 威生态系统红色名录》评估则借鉴了物种红色名录 的受威胁等级。由于每种生态系统类型的差异较大, 其结构与功能很难用同一指标衡量, 故该评估体系 主要利用生态系统面积变化来表征受威胁状况 (Noss et al, 1995)。基于生境面积、生态系统结构和 功能的评估方法为生态系统红色名录由理论研究 转向实际操作奠定了基础, 为IUCN生态系统红色 名录评估标准的制定积累了案例和经验。

虽然基于生境面积、生态系统结构和功能的评
估体系被许多国家采用，但仍然存在以下几个问题: (1)各评估案例的评价指标以及评估阈值不一致, 评 估结果之间的可比性较差; (2)各个评估案例中对生 态系统结构和功能的描述较为模糊, 实际上还是以 生境面积变化为主要评估依据; (3)仅用于局部地区, 很难应用于大区域生态系统的评估(Rodriguez et al, 2011; 朱超等, 2015)。为了解决上述问题, IUCN于 2008 年第 4 次世界自然保护大会上提出了构建 IUCN生态系统受威胁状况评估标准的想法。2009 年, 生态系统管理委员会(Commission on Ecosystem Management, CEM)将生态系统红色名录评估 纳入组委会重要议题, 并成立工作组, 开展IUCN 生态系统红色名录评估标准的制定工作(Rodríguez et al, 2012)。IUCN生态系统红色名录评估体系当前 已应用于全球50多个生态系统, 包括我国黄海滩涂 和辽河三角洲的评估 (陈国科和马克平, 2012; Murray et al, 2015), 其结果与当地的评估结果有较 好的一致性。IUCN生态系统红色名录评估框架以 较强的适用性、可执行性、系统性和科学性受到广 泛关注, 被IUCN采纳为评估生态系统受威胁状况 的正式标准(Keith et al, 2013; Rodríguez et al, 2015)。

\section{IUCN生态系统红色名录评估体系}

IUCN生态系统红色名录工作组在已有评估方 案的基础上, 从生态系统的生物组成、非生物环境、 相互作用以及空间分布4要素出发, 选取 5 个标准来 评估生态系统受威胁的风险: 生境范围退化(标准 A)、生境限制分布(标准B)、非生物环境退化(标准 C)、生物过程退化(标准D)、威胁定量分析(标准E) (Keith et al, 2013)。

生境范围退化(标准A)以生境面积在指定的时 间框架下的退化率来表征(公式(1))。根据物种与生 境面积的关系可知, 生态系统面积的减少会导致生 态位的宽度大幅度收缩, 降低生态系统维持生物多 样性的能力, 同时生态系统破碎化程度增加, 更容 易受干扰的影响, 使生态系统崩溃的风险增大 (Keith et al, 2013)。生态系统面积直接表征了生态系 统的承载力, 其退化率是指示生态系统受威胁的最 直观指标。该指标旨在确定生态系统面积退化的情 况, 并解析生境退化背后的驱动机制。为了解决实 际评估中数据时间跨度不足 50 年的情况, IUCN生 态系统红色名录工作组提出了在两期数据的时间 
跨度超过20年且面积变化规律与模型中变化规律 一致的情况下, 利用绝对变化量模型(absolute rate of decline, ARD) (公式(2)和(3))或绝对变化率模型 (proportional rate of decline, PRD) (公式(4)), 将变化 的时间跨度推广到50年(图1) (Rodríguez et al, 2015)。例如, 当仅有1990年和2010年的数据时, 评 估者可以利用该模型将变化率外推到过去 50 年 (1960-2010)和未来50年(1990-2040)。

$$
\begin{gathered}
\text { LOST }=\frac{\left(\text { Area }_{t 1}-\text { Area }_{t 2}\right)}{\text { Area }_{t 1}} \times 100 \% \\
\mathrm{ARD}=\frac{\left(\text { Area }_{t 2}-\text { Area }_{t 1}\right)}{\left(\text { year }_{t 2}-\text { year }_{t 1}\right) \times \text { Area }_{t 1}} \times 50 \\
\mathrm{ARD}=\frac{\left(\text { Area }_{t 2}-\text { Area }_{t 1}\right)}{\left(\text { year }_{t 2}-\text { year }_{t 1}\right) \times \text { Area }_{t 2}-50 \times\left(\text { Area }_{t 2}-\text { Area }_{t 1}\right)} \times 50 \\
\mathrm{PRD}=\left(\frac{\text { Area }_{t 2}}{\text { Area }_{t 1}}\right)^{\left(\frac{50}{\text { year }_{t 2}-\text { year }_{t 1}}\right)}-1
\end{gathered}
$$

式中, $A r e a_{t}$ 和 $y e a r_{t}$ 分别代表时间 $t$ 对应的面积和年 份。公式(2)和(3)分别代表ARD模型未来和过去50 年的外推公式, 公式(4)代表PRD模型中过去和未来 50年的外推公式。

生境限制分布(标准B)描述生态系统空间分布 的限制程度，表征威胁因子在生境斑块间的传播能 力以及生境的承载力(Keith et al, 2013)。借鉴物种红 色名录标准, 标准B采用生境范围(extent of occurrence, EOO)、生境所占面积(area of occupancy, AOO)
和生境斑块数目表征(Keith et al, 2013)。EOO指生态 系统的分布范围, 用生态系统所有生境组成的最小 外接凸包多边形表示(图2), 该指标主要表征生态系 统传播风险的能力。AOO指生态系统占据的面积大 小, 用生态系统占据 $10 \mathrm{~km} \times 10 \mathrm{~km}$ 的有效网格数目 来表示(有效网格指生态系统面积大于网格面积 $1 \%$ 的网格) (图2)。该指标主要表征适宜生境的大小。 生境斑块数目指有限且离散的生境斑块的数目。标 准 $\mathrm{B}$ 在实际评估过程中需满足生境斑块限制分布或 者生境有持续退化的趋势(Rodríguez et al, 2015)。

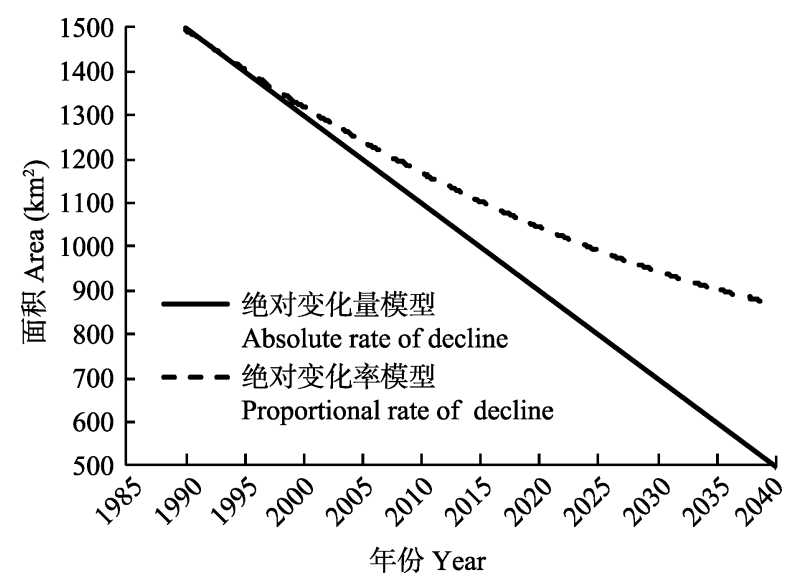

图1 绝对变化量模型和绝对变化率模型预测结果对比(改 编自Rodríguez et al, 2015)

Fig. 1 Results derived from proportional rate of decline versus absolute rate of decline (adapted from Rodríguez et al, 2015)

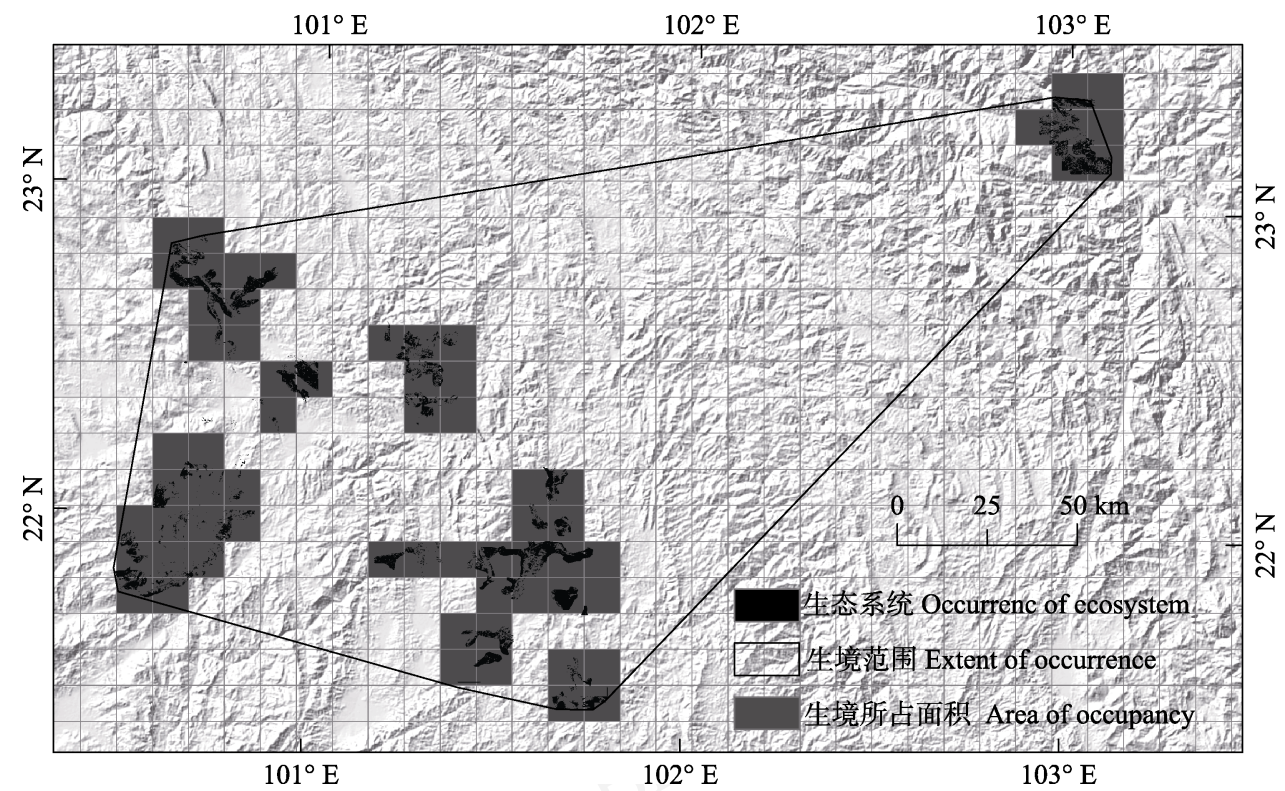

图2 生境限制分布(标准B)示意图。生境范围(EOO)为 $28,249 \mathbf{k m}^{2}$, 生境所占网格数目(AOO)为 88(改编自Keith et al, 2013)。 Fig. 2 The diagram of habitat restricted distribution (Criterion B). Extent of Occurrence (EOO) is 28,249 $\mathrm{km}^{2}$ and Area of Occupancy (AOO) is 88 (adapted from Keith et al, 2013). 
非生物环境退化(标准C)和生物过程退化(标准 D)分别表征生态系统的环境要素和生物过程对威 胁的响应。标准C用生态系统关键的非生物环境要 素的退化程度来表征。生态系统都依赖于特定的环 境而存在, 当非生物环境发生退化时, 环境提供给 生态系统的物质和能量减少, 导致生态系统维持生 物多样性的能力降低, 生态系统受威胁的风险增加 (Keith et al, 2013)。随着人类活动不断加剧以及全球 变化等环境问题的日益突出(Vitousek et al, 1997), 该指标在评估过程中的作用愈发明显。标准D用表 征生物间相互作用的关键变量的退化情况来描述。 生态系统中物质、能量和信息的循环都需要通过生 物体间的相互作用维持, 包括捕食、授粉、互利共 生等(Fontaine et al, 2005)。当其中一个环节出现断 裂, 必然影响生态系统的正常运行, 增大崩溃的风 险(Mitchell et al, 2006)。一般来说, 生态系统的生物 多样性水平越高, 其生物过程越稳定、复杂, 能量 和信息的流动也越通畅, 表现为更强的获取能量资 源的能力、更快的物质能量循环速度和更稳定的生 态系统(Loreau, 2010; Keith et al, 2013)。实际评估过 程中, 首先需明确直接决定区域生态系统状况的非 生物环境以及生物过程的相关变量(Rodriguez et al, 2015), 然后利用公式(5)和(6)获取生态系统受胁迫 的严重程度(relative severity) 以及退化程度(extent of degradation), 从而获取生态系统的衰退情况。其 中, 受胁迫的严重程度、退化程度分别描述了生态 系统接近崩溃的程度以及威胁影响的范围。

$$
\text { relative severity }=\frac{\left(\mathrm{Var}_{t}-\text { initial }\right)}{\text { collapse }- \text { initial }} \times 100 \%
$$

extent of degradation $=\frac{\text { distribution } \text { affected by decline }}{\text { total distribution }} \times 100 \%(6)$ 式中, $V r_{t}$ 代表时间 $t$ 下非生物环境或者生物过程相 关变量的值; initial和collapse分别代表生态系统初 始状态 (无威胁) 和崩溃状态下相关变量的值 (Rodriguez et al, 2015)。

威胁定量分析(标准E)指用模型定量描述生态 系统以及干扰过程, 模拟生态系统对威胁的响应, 并结合威胁的发展情景综合评估生态系统崩溃的 风险(Bland et al, 2015)。由于生态系统本身的复杂 性以及威胁的多样性, 精细表征各生态系统干扰过 程的模型仍严重缺乏, 该标准在实际评估过程中应 用较少(Boitani et al, 2015)。

IUCN生态系统红色名录评估流程见图3。首先 根据可获取的数据得到以上 5 个标准的相关变量。 然后结合评估体系中各个评估标准的阈值, 将其分 为以下等级: 无威胁类别 2 种, 分别为无危 (Least Concern, LC)和近危(Near Threatened, NT); 受威胁 类别3种, 分别为易危(Vulnerable)、濒危(Endangered, EN)和极危(Critically Risk, CR), 其中极危的威胁程 度最高, 无危最低。最后选取各标准中表征生态系 统受威胁程度最高的等级作为该生态系统最终的威 胁状况(最大风险原则)。对于受到严重威胁的生态 系统, 无法恢复到原来状况的则列为崩溃(Collapsed, CO) (Rodríguez et al, 2015)。各标准中, 标准A 和标准 $\mathrm{B}$ 与生态系统面积和分布相关, 由于此类空 间数据相对于其他类型数据的可获得性更高, 因此 这两个标准是评估中最常用的指标(Boitani et al, 2015)。

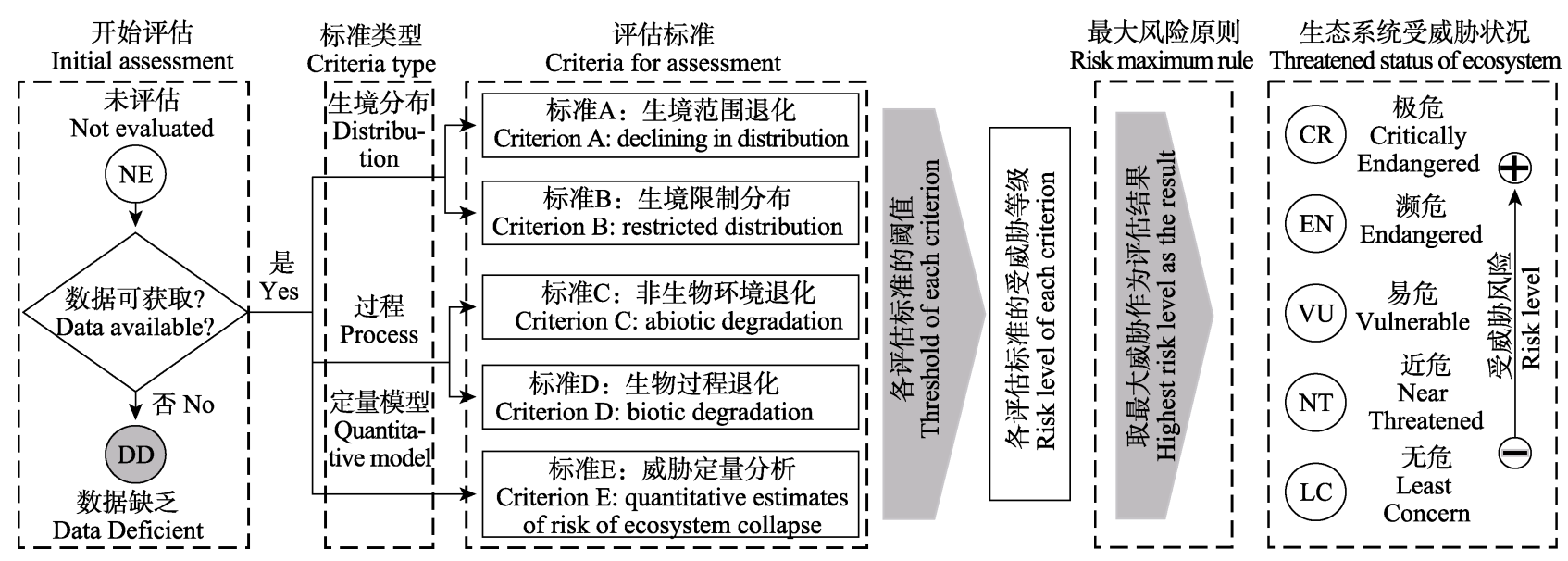

图3 IUCN生态系统受威胁状况评估流程(改编自Rodríguez et al, 2015)

Fig. 3 The steps followed for the application of IUCN of Ecosystems Categories and Criteria (adapted from Rodríguez et al, 2015) 
相较于以往的生态系统受威胁状况评估方法, 该评估体系重点突出了生态系统评估对象以及生 态系统崩溃等基本概念, 有利于加深评估者对生态 系统受威胁状况评估的认识。由于缺乏数据基础以 及生态系统本身的复杂性, 衡量标准 $\mathrm{C}$ 和标准 $\mathrm{D}$ 的 指标更多地处于概念和模型设计阶段, 还有待进一 步研究(Boitani et al, 2015)。该评估体系采用风险最 大的原则, 在部分指标当前无法获取的情况下, 依 然可以开展评估工作, 从而最大限度地保证了评估 体系的通用性(Keith et al, 2015)。

\section{IUCN生态系统红色名录评估面临的挑战}

当前IUCN生态系统红色名录评估标准及相应 阈值的设定缺乏理论和数据的支撑, 在生态系统制 图和生态系统崩溃的概念以及实际操作层面均不 够清晰, 易导致在应用时主观地解读评估体系, 形 成不确定的结果, 这是当前IUCN生态系统红色名 录实际评估过程中面临的主要挑战(Boitani et al, 2015)。

\section{1 生态系统制图及其不确定性}

生态系统红色名录评估首先需明确评估对象 及其时空分布, 即生态系统制图。通过生态系统制 图, 可确定各生态系统的边界, 获取评估单元(Jones et al, 1994), 是开展生态系统红色名录评估工作的 前提和基础。IUCN生态系统红色名录评估体系中, 土地覆被类型图、生态功能区划图以及植被图均是 生态系统制图的重要数据源(Olson et al, 2001; Keith et al, 2013; Payet et al, 2013)。然而, 因生态系统的 划分方法各异, 而各地方数据不齐全, 导致评估体 系的适用性和评估结果的可比性差。同时, 由于缺 乏普遍接受的生态系统分类体系, 导致评估单元不 明确, 在应用该评估体系的过程中, 容易主观地利 用相关变量来表征评估体系的标准, 造成结果的不 确定性。由于评估的目的和可用的数据不一致, 加 上生态系统本身的巢式结构的特点, 构建一套等级 层次的生态系统分类系统, 以满足不同区域、不同 层次的生态系统红色名录评估, 是解决生态系统制 图不一致性的关键。遥感作为宏观尺度上对地观测 的重要手段, 因其多尺度的观测特点以及不断增强 的地物识别能力, 使之成为生态系统制图最具潜力 的手段(Pettorelli et al, 2014; de Araujo Barbosa et al, 2015)。因此, 如何完善生态系统分类体系, 构建基
于遥感的生态系统分类方法, 是建立全球生态系统 红色名录的前提(Keith et al, 2013; Sayre et al, 2014; Nicholson et al, 2015)。

在我国生态系统受威胁状况评估中, 潜在的 可用数据主要包括 $1: 100$ 万植被图和 $30 \mathrm{~m}$ 空间分辨 率的土地覆被图。1:100万植被图(中国科学院中国 植被图编辑委员会, 2007)是我国三代科学家耗费 近30年的时间，通过大量的实地调查和判读得到 的, 具有丰富的植被类型信息, 但空间定位精度和 动态变化信息略显不足。土地覆被图是基于遥感影 像采用自动或人工解译得到的, 具有动态变化信 息以及较高的空间位置精度, 但其包含的类别信 息较少，例如ChinaCover产品仅有38个类别 (Lei et al, 2016), 中国土地利用数据仅有25个类别(刘纪 远, 1997)。因此, 融合这两种数据类型, 发挥各自 优势, 实现高时空分辨率的生态系统制图, 可以为 在全国尺度上开展生态系统红色名录的评估提供 数据基础。

\section{2 生态系统崩溃的定义及其表征}

生态系统崩溃不能完全与物种灭绝类比(Boitani et al, 2015)。如果确定某一物种的最后一个个体已 经死亡, 则认为该物种已灭绝(IUCN SSC, 2001)。然 而, 一个生态系统崩溃时, 其所处的生境可能并没 有完全消失, 但原来的服务功能退化、关键特征缺 失或已转化为新的生态系统类型(Keith et al, 2013), 这导致生态系统崩溃的定义难以界定(Crespin \& Simonetti, 2015)。

生态系统崩溃主要是由于生物链的断层、物质 循环和能量流动的停滞、生物间相互作用和非生物 环境的剧烈变化, 导致生态系统难以维持原有的生 物功能或过程(Crespin \& Simonetti, 2015)。弄清楚 生态系统崩溃的过程是了解生态系统退化机制、确 定生态系统红色名录中指标以及阈值的基础, 是开 展针对性的保护和管理的前提, 对于规范IUCN生 态系统红色名录评估体系的应用至关重要。IUCN 生态系统红色名录评估框架中通过类比物种红色 名录, 利用图3中的 5 个标准来衡量一个生态系统的 崩溃风险(Boitani et al, 2015)。然而, 全球生态系统 类型众多, 威胁因素也多样化, 各个生态系统的过 程和机制均差异较大, 对威胁的响应不一致, 基于 这5个指标能否全面表征生态系统的崩溃风险还需 进一步研究(Boitani et al, 2015)。 


\section{3 评估尺度及其效应}

生态系统是基于一定时空尺度定义的，具有明 显的尺度依赖性(Wiens, 1989; Begon et al, 2005)。在 不同尺度上, 同一生态系统对相同威胁的响应也不 一样, 这将直接影响评估结果(Keith, 2009; Keith et $\mathrm{al}, 2013)$ 。生态系统红色名录评估中的尺度问题归 根结底是生态系统尺度效应的具体体现, 主要包括 时间尺度、空间尺度、分类等级尺度3个方面(Keith, 2009)。

IUCN生态系统红色名录评估体系中, 生境退 化率、非生物环境退化以及生物过程退化等均是在 一定的时间跨度上计算的, 不同的时间尺度直接影 响评估结果(Keith et al, 2013)。生态系统在一定的时 间段内表现出较高的稳定性, 但受到干扰时, 生态 系统在该时间尺度下将发生变化。评估过程中, 由 于生态系统对威胁有一定的适应能力(Rapport et al, 1985), 且生态系统本身也有正常的自然波动(Keith et al, 2013)。因此, 评估的时间尺度必须能反映生态 系统过去的状况和未来的变化趋势, 同时能区分出 生态系统正常的自然动态。IUCN生态系统红色名 录评估体系中采用 “过去50年”、“未来50年” 以及 “1750年以来”作为相应标准的时间尺度(Rodríguez et al, 2015)。然而, 这种时间尺度划分方式的科学依 据、数据基础以及基于该时间尺度的评估结果对保 护决策的影响仍不明确(Boitani et al, 2015)。同时, 由于较长的时间跨度易导致可用数据不足, 采用 ARD和PRD预测模型很难满足所有退化情景模式。 利用遥感以及生态系统分布与气候关系模型等技 术获取长时间序列数据, 结合土地覆被驱动力分析 和预测模型, 精细表征生态系统变化过程, 是解决 该问题的途径之一。

空间尺度主要包括评估的空间范围以及划分 生态系统的空间粒度。一般来说, 评估的范围越大, 生态系统分布越广泛, 受威胁程度也越低(Wiens, 1989)。因此, 不同评估范围可能产生不同的评估结 果(Keith, 2009)。在实际评估中, 为了方便科学管理, 可以结合评估的目的, 选择国家尺度、行政单元开 展评估, 同时还需考虑生态系统在评估范围内的空 间代表性, 保证评估结果的一致性和合理性。空间 粒度是生态系统最小可辨识的空间单元(Wiens, 1989), 划分生态系统的网格可以小到仅包括一棵 树, 大到包括整个生态系统。一般情况下，一个粒
度单元内可能包含多种生态系统, 但仅能表示出一 种生态系统, 粒度越大, 网格内主要生态系统越凸 显, 而非主导的生态系统则被忽略, 从而改变各生 态系统的生境面积(Turner et al, 1989), 最终影响评 估结果。由于许多濒危生态系统的生境狭小, 过大 的粒度容易忽略其较小的生境, 造成生态系统受威 胁的风险被高估; 而对于宏观的生态系统, 过小的 粒度往往显著增加了数据量和计算的复杂性。在实 际评估中, 需根据评估目的以及可用的数据, 确定 评估过程的最佳空间尺度, 尽量减少尺度效应带来 的不确定性(Boitani et al, 2015)。

生态系统分类体系是一个等级系统(O’Neill, 1986; Klijn \& de Haes, 1994), 分类等级尺度就是确 定评估对象在分类等级(如植被型、群系、群丛)中 的位置(如群系) (Keith, 2009)。IUCN生态系统红色 名录评估中, 分类等级尺度是确定评估单元以及生 态系统功能和过程的基础。同一地区不同的分类等 级尺度对应不同层次的评估对象, 所表现的生物过 程也不尽相同, 这些都会直接影响评估结果 (Peterson et al, 1998)。较大的分类等级尺度对应宏 观的生态系统, 其粒度也应该更粗, 评估过程较宏 观; 反之, 较小的分类等级尺度, 其对数据粒度的 要求更精细。实际评估过程中, 需要明确评估对象 以及可获取的数据, 确定评估中生态系统的分类等 级尺度, 但解析不同的分类等级尺度对评估结果的 影响是确定分类等级尺度的前提。

评估过程中, 不同的尺度会得到不同的评估结 果, 同时不同尺度类型之间也相互作用, 但尺度效 应对评估结果的作用有多大, 哪些因素与尺度效应 密切相关, 不同尺度类型之间的定量关系, 以及在 实际评估中如何根据有限的数据确定最佳的评估 尺度, 仍缺乏相关研究。

\section{4 评估标准存在的不足及解决方案}

随着IUCN生态系统红色名录评估标准在实际 评估工作中的运用, 其评估标准面临的挑战也逐渐 显现, 如: 评估标准的阈值缺乏理论基础、EOO的 科学性不足、难以确定生物过程和非生物环境相关 变量、评估结果缺乏生境退化的空间信息等。

评估中如何确定评估阈值是评估工作的重要 内容, 也是研究的难点。理想的情况是利用相关的 评估指标与原始健康生态系统进行对比, 结合生态 系统崩溃的状态, 来衡量被评估生态系统的受威胁 
和健康程度，确定评估的阈值 (Rapport，1989; Rodríguez et al, 2015)。IUCN生态系统红色名录评估 标准参考物种红色名录评估体系, 在评估过程中均 采用同一套阈值(Boitani et al, 2015)。然而实际情况 是生态系统面临的威胁因素多样, 且各生态系统类 型对威胁的响应不一致, 评估指标的阈值与生态系 统类型和受威胁因素相关; 部分评估指标具有尺度 依赖性, 其阈值大小与指标的尺度水平相关, 同一 生态系统在不同尺度下的评估结果差异较大。

IUCN生态系统红色名录评估体系参考物种红 色名录标准, 利用生态系统所占据的最小外接凸包 多边形的面积(EOO)作为评估风险传播能力的一个 指标。该指标越大, 则表明斑块间距离越远, 风险 传播的能力越弱, 该类生态系统受威胁的程度越低 (Keith et al, 2013)。而在景观生态学以及岛屿生物地 理学理论中, 同类型的斑块间距越大, 生境越离散, 生境隔离程度越大, 生态系统越不稳定, 斑块中物 种灭绝的风险越高(Saunders et al, 1991; Collinge, 1996; Fahrig, 2003)。物种红色名录评估体系中, 研 究者也逐渐意识到这个问题, 建议排除EOO中不适 合物种分布的区域(Mace et al, 2008), 但IUCN生态 系统红色名录评估标准中并没有考虑这一点, 导致 该指标表征的威胁程度可能偏低(Ostro et al, 1999; IUCN, 2016)。

生物过程和非生物环境退化描述了来自生态 系统功能、服务衰退和生态系统所处环境恶化的威 胁, 是生态系统受威胁状况的关键指标(Vázquez \& Simberloff, 2003)。然而, 当前的评估案例中仅简单 地对少数几个生物过程和非生物环境退化明显的 生态系统进行了评估(Keith et al, 2013)。对于复杂以 及大范围区域, 由于各生态系统生物过程和非生物 环境的异质性以及复杂性, 生物过程和非生物环境 与生态系统的相互作用不清楚, 难以获取直接表征 生态系统状况的生物过程和非生物环境相关变量, 该标准在实际评估中应用的难度较大(Boitani et al, 2015)。

IUCN生态系统红色名录评估的目的在于针对 有限的保护资源确定保护优先区域, 指导生物多样 性保护决策, 确定生态系统退化的威胁因素。然而 在复杂地区, 同一生态系统的生境斑块在空间上的 退化程度往往有所差异, 而IUCN生态系统红色名
录评估的结果仅包括受威胁生态系统的目录, 缺乏 各生境斑块退化的空间位置信息。生态系统限制分 布指标能指示生态系统的空间分布，但不足以表征 该类生态系统各斑块退化的空间位置信息。而这一 信息在生态系统离散分布以及有限的保护资源情 况下, 对于有针对性地开展生态系统管理和维护工 作显得极为重要(Christensen et al, 1996)。采用等级 尺度评估方法, 利用精细尺度下的评估结果弥补宏 观尺度的不足，丰富生态系统红色名录空间退化信 息，是解决该问题的途径之一。

\section{IUCN生态系统红色名录发展趋势与展望}

尽管IUCN生态系统红色名录评估框架在实际 评估中仍然面临较大挑战, 但该评估体系从生态系 统组成要素的受威胁状况出发, 直观地开展风险评 估, 为生态系统的评估提供了更为直接、客观的方 法体系(Keith et al, 2015)。在生物多样性评估与保护 以及不断丰富和探索该评估标准的迫切需求下, 生 态学家以及生物保护学家已基于该标准在全球开 展了相关的评估工作(Keith et al, 2015)。

地面试验和观测是IUCN生态系统红色名录评 估的重要手段之一, 而随着研究的广度以及动态程 度不断提高, 开展区域乃至全球尺度生态系统动态 监测的研究不断涌现, 利用遥感以及生态系统模型 结合地面试验开展生态系统的评估成为研究的热 点。遥感本身固有的特点使其在生态系统实时监测 以及尺度效应分析方面有着其他手段无法替代的 优势(Wang et al, 2010; Pettorelli et al, 2016), 而生态 系统模型是描述生物过程以及生态系统环境响应 的重要手段。主要体现在: (1)利用遥感宏观的观测 能力, 以及多源、高时空和高光谱等特点，是获取 长时间序列、精细的生态系统制图的重要数据源之 一; (2)不同尺度下的生态系统红色名录评估结果差 异巨大, 对比不同尺度下的评估结果, 解析尺度效 应对评估的影响, 是今后研究的热点; (3)生物过程 以及非生物环境指标作为IUCN生态系统红色名录 评估体系中的重要指标, 由于缺乏数据基础, 其实 际可操作性不高, 随着对生态系统的观测和模型模 拟愈加精细, 结合现有多种观测手段, 对生态系统 实时监测, 提取各生态系统关键变量, 对该指标进 行直观表达, 是未来研究的一个重要方向。 
根据IUCN生态系统红色名录发展趋势可以看 出, 融合多学科以及多种手段研究生态系统中的相 关问题, 完善生态系统红色名录评估体系是今后研 究的重点和关键。随着遥感多源多尺度以及星-机地协同观测能力的增强, 以及更加科学的生态系统 模型不断涌现，耦合多种技术手段协同监测生态系 统, 为IUCN生态系统红色名录评估中部分问题的 解决以及实现全球评估提供了新的契机。

\section{参考文献}

Barrett GW (1978) Stress effects on natural ecosystems. Ohio Journal of Science, 78, 160-162.

Begon M, Townsend CR, Harper JL (2005) Ecology: From Individuals to Ecosystems, 4th edn. Blackwell Publishing, Oxford.

Blab J, Riecken U, Ssymank A (1995) Proposal on a criteria system for a National Red Data Book of Biotopes. Landscape Ecology, 10, 41-50.

Bland LM, Keith DA, Murray NJ, Rodriguez JP (2015) Guidelines for the application of IUCN Red List of Ecosystems Categories and Criteria. http://iucnrle.org/static/media/uploads/references/key\%20documents/bland-etal-2016_iucn_rle_ guidelines_v1.pdf/. (accessed 2016-03-14)

Boitani L, Mace GM, Rondinini C (2015) Challenging the scientific foundations for an IUCN Red List of Ecosystems. Conservation Letters, 8, 125-131.

Burgess ND, Hales JD, Ricketts TH, Dinerstein E (2006) Factoring species, non-species values and threats into biodiversity prioritisation across the ecoregions of Africa and its islands. Biological Conservation, 127, 383-401.

Cardinale BJ, Duffy JE, Gonzalez A, Hooper DU, Perrings C, Venail P, Narwani A, Mace GM, Tilman D, Wardle DA (2012) Biodiversity loss and its impact on humanity. Nature, 486, 59-67.

Ceballos G, Ehrlich PR, Barnosky AD, García A, Pringle RM, Palmer TM (2015) Accelerated modern human-induced species losses: entering the sixth mass extinction. Science Advances, 1, e1400253.

Chen GK, Ma KP (2012) Criteria and methods for assessing the threat status of ecosystem. Biodiversity Science, 20, 66-75. (in Chinese with English abstract) [陈国科, 马克平 (2012) 生态系统受威胁等级的评估标准和方法. 生物多样性, 20, 66-75.]

Editorial Committee of Vegetation Atlas of China, Chinese Academy of Sciences (2007) 1: 1000000 Vegetation Atlas of People's Republic of China. Geological Publishing House, Beijing. (in Chinese) [中国科学院中国植被图编辑 委员会 (2007) 中华人民共和植被图(1: 1000000). 地质 出版社, 北京. ]

Christensen NL, Bartuska AM, Brown JH, Carpenter S,
D’Antonio C, Francis R, Franklin JF, MacMahon JA, Noss RF, Parsons DJ, Peterson CH, Turner MG, Woodmansee RG (1996) The report of the Ecological Society of America Committee on the scientific basis for ecosystem management. Ecological Applications, 6, 665-691.

Collinge SK (1996) Ecological consequences of habitat fragmentation: implications for landscape architecture and planning. Landscape and Urban Planning, 36, 59-77.

Commonwealth of Australia (1999) Environment Protection and Biodiversity Conservation Act. Department of Environment, Australian. https://www.legislation.gov.au/Details/ C2016C00431. (accessed 2016-02-08)

Crespin SJ, Simonetti JA (2015) Predicting ecosystem collapse: spatial factors that influence risks to tropical ecosystems. Austral Ecology, 40, 492-501.

de Araujo Barbosa CC, Atkinson PM, Dearing JA (2015) Remote sensing of ecosystem services: a systematic review. Ecological Indicators, 52, 430-443.

Driver A (2005) National Spatial Biodiversity Assessment 2004: Priorities for Biodiversity Conservation in South Africa. PhD disseration, National Biodiversity Institute, South Africa.

Fahrig L (2003) Effects of habitat fragmentation on biodiversity. Annual Review of Ecology, Evolution, and Systematics, 34, 487-515.

Ferrier S (2002) Mapping spatial pattern in biodiversity for regional conservation planning: where to from here? Systematic Biology, 51, 331-363.

Fontaine C, Dajoz I, Meriguet J, Loreau M (2005) Functional diversity of plant-pollinator interaction webs enhances the persistence of plant communities. PLoS Biology, 4, e1.

Hector A, Bagchi R (2007) Biodiversity and ecosystem multifunctionality. Nature, 448, 188-190.

Hooper DU, Chapin IF, Ewel JJ, Hector A, Inchausti P, Lavorel S, Lawton JH, Lodge DM, Loreau M, Naeem S (2005) Effects of biodiversity on ecosystem functioning: a consensus of current knowledge. Ecological Monographs, 75, 3-35.

IUCN Species Survival Commission (SSC) (2001) IUCN Red List Categories and Criteria, Version 3.0. IUCN Publication, Switzerland.

IUCN (2016) Guidelines for using the IUCN Red List Cat egories and Criteria, Version 12.0. http://www.iucnredlis t.org/documents/RedListGuidelines.pdf/. (accessed 2016-0 1-05)

Jones CG, Lawton JH, Shachak M (1994) Organisms as Ecosystem Engineers, pp. 130-147. Springer, New York.

Keith DA (2009) The interpretation, assessment and conservation of ecological communities. Ecological Management \& Restoration, 10, S3-S15.

Keith DA (2015) Assessing and managing risks to ecosystem biodiversity. Austral Ecology, 40, 337-346.

Keith DA, Rodríguez JP, Brooks TM, Burgman MA, Barrow 
EG, Bland L, Comer PJ, Franklin J, Link J, McCarthy MA (2015) The IUCN Red List of Ecosystems: motivations, challenges and applications. Conservation Letters, 8, 214226.

Keith DA, Rodríguez JP, Rodríguez-Clark KM, Nicholson E, Aapala K, Alonso A, Asmussen M, Bachman S, Basset A, Barrow EG (2013) Scientific foundations for an IUCN Red List of Ecosystems. PLoS ONE, 8, e62111.

Klijn F, de Haes HAU (1994) A hierarchical approach to ecosystems and its implications for ecological land classification. Landscape Ecology, 9, 89-104.

Lackey RT (1994) Ecological risk assessment. Fisheries, 19, 14-19.

Lei GB, Li AN, Bian JH, Zhang ZJ, Jin HA, Nan X, Zhao W, Wang JY, Cao XM, Tan JB, Liu QN, Yu H, Yang GB, Feng WL (2016) Land cover mapping in southwestern China using the HC-MMK approach. Remote Sensing, 8, 1-22.

Lindgaard A, Henriksen S (2011) The 2011 Norwegian Red List for ecosystems and habitat types. Norwegian Biodiversity Information Centre, Trondheim. http://www.artsdatabanken.no/Files/13976/Norwegian_Red_List_for_Ecosyst ems_and_Habitat_Types_2011_(PDF)/. (accessed on 201603-14)

Liu JY (1997) Study on national resources \& environment survey and dynamic monitoring using remote sensing. Journal of Remote Sensing, 1, 225-230. (in Chinese with English abstract) [刘纪远 (1997) 国家资源环境遥感宏观 调查与动态监测研究. 遥感学报, 1, 225-230.]

Loreau M (2010) Linking biodiversity and ecosystems: towards a unifying ecological theory. Philosophical Transactions of the Royal Society B: Biological Sciences, 365, 49-60.

Ma KP (2013) Studies on biodiversity and ecosystem function via manipulation experiments. Biodiversity Science, 21, 247-248. (in Chinese) [马克平 (2013) 生物多样性与生态 系统功能的实验研究. 生物多样性, 21, 247-248.]

Ma KP (2016) Hot topics for biodiversity science. Biodiversity Science, 24, 1-2. (in Chinese) [马克平 (2016) 生物多样性 科学的热点问题. 生物多样性, 24, 1-2.]

Ma KP, Qian YQ (1998) Biodiversity conservation and its research progress. Chinese Journal of Applied and Environmental Biology, 4, 95-99. (in Chinese with English abstract) [马克平, 钱迎倩 (1998) 生物多样性保护及其 研究进展. 应用与环境生物学报, 4, 95-99.]

MA (Millennium Ecosystem Assessment) (2005) Ecosystems and Human Well-being: Biodiversity Synthesis. Island Press, Washington, DC.

Mace GM, Collar NJ, Gaston KJ, Hilton-Taylor C, Akçakaya R, Leader-Williams N, Milner-Gulland EJ, Stuart SN (2008) Quantification of extinction risk: IUCN's system for classifying threatened species. Conservation Biology, 22, 1424-1442.

Mitchell CE, Agrawal AA, Bever JD, Gilbert GS, Hufbauer RA, Klironomos JN, Maron JL, Morris WF, Parker IM,
Power AG, Seabloom EW, Torchin ME, Vazquez DP (2006) Biotic interactions and plant invasions. Ecology Letters, 9, 726-740.

Murray NJ, Ma ZJ, Fuller RA (2015) Tidal flats of the Yellow Sea: a review of ecosystem status and anthropogenic threats. Austral Ecology, 40, 472-481.

Myers N, Mittermeier RA, Mittermeier CG, da Fonseca GAB, Kent J (2000) Biodiversity hotspots for conservation priorities. Nature, 403, 853-858.

Nicholson E, Keith DA, Wilcove DS (2009) Assessing the threat status of ecological communities. Conservation Biology, 23, 259-274.

Nicholson E, Regan TJ, Auld TD, Burns EL, Chisholm LA, English V, Harris S, Harrison P, Kingsford RT, Leishman MR, Metcalfe DJ, Pisanu P, Watson CJ, White M, White M, Williams RJ, Wilson B, Keith DA (2015) Towards consistency, rigour and compatibility of risk assessments for ecosystems and ecological communities. Austral Ecology, 40, 347-363.

Noss RF (1990) Indicators for monitoring biodiversity: a hierarchical approach. Conservation Biology, 4, 355-364.

Noss RF (1996) Ecosystems as conservation targets. Trends in Ecology \& Evolution, 11, 419-422.

Noss RF, LaRoe ET, Scott JM (1995) Endangered ecosystems of the United States: a preliminary assessment of loss and degradation. US Department of the Interior, National Biological Service, Washington, DC.

O’Neill RV (1986) A Hierarchical Concept of Ecosystems. Princeton University Press, Princeton.

Odum EP (1977) The emergence of ecology as a new integrative discipline. Science, 195, 1289-1293.

Odum EP (1985) Trends expected in stressed ecosystems. BioScience, 35, 419-422.

Olson DM, Dinerstein E, Wikramanayake ED, Burgess ND, Powell GVN, Underwood EC, D’amico JA, Itoua I, Strand HE, Morrison JC, Loucks CJ, Allnutt TF, Ricketts TH, Kura Y, Lamoreux JF, Wettengel WW, Hedao P, Kassem KR (2001) Terrestrial ecoregions of the world: a new map of life on earth. BioScience, 51, 933-938.

Ostro LET, Young TP, Silver SC, Koontz FW (1999) A geographic information system method for estimating home range size. Journal of Wildlife Management, 63,748-755.

Payet K, Rouget M, Esler KJ, Reyers B, Rebelo T, Thompson MW, Vlok JHJ (2013) Effect of land cover and ecosystem mapping on ecosystem-risk assessment in the little karoo, South Africa. Conservation Biology, 27, 531-541.

Peterson G, Allen CR, Holling CS (1998) Ecological resilience, biodiversity, and scale. Ecosystems, 1, 6-18.

Pettorelli N, Laurance WiF, O’Brien TG, Wegmann M, Nagendra H, Turner W (2014) Satellite remote sensing for applied ecologists: opportunities and challenges. Journal of Applied Ecology, 51, 839-848.

Pettorelli N, Wegmann M, Skidmore A, Mücher S, Dawson 
TP, Fernandez M, Lucas R, Schaepman ME, Wang T, O’Connor B (2016) Framing the concept of satellite remote sensing essential biodiversity variables: challenges and future directions. Remote Sensing in Ecology and Conservation, 2, 122-131.

Pimm SL, Jenkins CN, Abell Robin, Brooks TM, Gittleman JL, Joppa LN, Raven PH, Roberts CM, Sexton JO (2014) The biodiversity of species and their rates of extinction, distribution, and protection. Science, 344, 1246752.

Pimm SL, Russell GJ, Gittleman JL, Brooks TM (1995) The future of biodiversity. Science, 269, 347-350.

Rapport DJ (1989) What constitutes ecosystem health? Perspectives in Biology and Medicine, 33, 120-132.

Rapport DJ, Regier HA, Hutchinson TC (1985) Ecosystem behavior under stress. The American Naturalist, 125, 617640.

Rapport DJ, Costanza Robert, McMichael AJ (1998) Assessing ecosystem health. Trends in Ecology \& Evolution, 13, 397-402.

Rodríguez JP, Balch JK, Rodríguez-Clark KM (2006) Assessing extinction risk in the absence of species-level data: quantitative criteria for terrestrial ecosystems. Biodiversity and Conservation, 16, 183-209.

Rodríguez JP, Keith DA, Rodríguez-Clark KM, Murray NiJ, Nicholson E, Regan TJ, Miller RM, Barrow EG, Bland LM, Boe K, Brooks TM, Oliveira-Miranda MA, Spalding M, Wit P (2015) A practical guide to the application of the IUCN Red List of Ecosystems criteria. Philosophical Transactions of the Royal Society B: Biological Sciences, 370, 1-9.

Rodríguez JP, Rodríguez-Clark KM, Keith DA, Barrow EG, Benson J, Nicholson E, Wit P (2012) IUCN red list of ecosystems. Surveys and Perspectives Integrating Environment and Society, 5, 61-70.

Rodríguez JP, Rodriguez-Clark KM, Baillie JE, Ash N, Benson J, Boucher T, Brown C, Burgess ND, Collen B, Jennings M, Keith DA, Nicholson E, Revenga C, Reyers B, Rouget M, Smith T, Spalding M, Taber A, Walpole M, Zager I, Zamin T (2011) Establishing IUCN Red List criteria for threatened ecosystems. Conservation Biology, 25, 21-29.

Rouget M, Reyers B, Jonas Z, Desmet P, Driver A, Maze K,
Egoh B, Cowling RM, Mucina L, Rutherford MC (2004) South African National Spatial Biodiversity Assessment 2004: Technical Report. Vol. 1. Terrestrial component. South African National Biodiversity Institute, Pretoria.

Saunders DA, Hobbs Richard J, Margules Chris R (1991) Biological consequences of ecosystem fragmentation: a review. Conservation Biology, 5, 18-32.

Sayre R, Dangermond J, Frye C, Vaughan R, Aniello P, Breyer S, Cribbs D, Hopkins D, Nauman R, Derrenbacher W (2014) A New Map of Global Ecological Land Units-An Ecophysiographic Stratification Approach. Association of American Geographers, Washington, DC. http://www.aag. org/galleries/default-file/AAG_Global_Ecosyst_bklt72.pdf/. (accessed 2016-02-08)

Turner MG, O’Neill RV, Gardner RH, Milne BT (1989) Effects of changing spatial scale on the analysis of landscape pattern. Landscape Ecology, 3, 153-162.

Vázquez DP, Simberloff D (2003) Changes in interaction biodiversity induced by an introduced ungulate. Ecology Letters, 6, 1077-1083.

Vitousek PM, Mooney HA, Lubchenco J, Melillo JM (1997) Human domination of Earth's ecosystems. Science, 277, 494-499.

Walker S, Price R, Rutledge D, Stephens RTT, Lee WG (2006) Recent loss of indigenous cover in New Zealand. New Zealand Journal of Ecology, 30, 169-177.

Wang K, Franklin SE, Guo XL, Cattet M (2010) Remote sensing of ecology, biodiversity and conservation: a review from the perspective of remote sensing specialists. Sensors, 10, 9647-9667.

Wiens JA (1989) Spatial scaling in ecology. Functional Ecology, 3, 385-397.

Zhu C, Fang Y, Zhou KX, Mu SJ, Jiang JL (2015) IUCN Red List of Ecosystems, a new tool for biodiversity conservation. Acta Ecologica Sinica, 35, 2826-2836. (in Chinese with English abstract) [朱超, 方颖, 周可新, 穆少杰, 蒋金亮 (2015) 生态系统红色名录:一种新的生物多样性保护工 具. 生态学报, 35, 2826-2836.]

(责任编委: 薛达元 责任编辑: 黄祥忠) 